\title{
IMAGING
}

\section{Performance of multidetector computed tomography colonography compared with conventional colonoscopy}

\author{
Th Gluecker, G Dorta, W Keller, P Jornod, R Meuli, P Schnyder
}

Gut 2002;51:207-211

See end of article for authors' affiliations

......................

Correspondence to:

Dr P Schnyder, Department

of Diagnostic and

Interventional Radiology,

University Hospital, CHUV,

1011 Lausanne,

Switzerland;

Pierre.Schnyder@

chuv.hospvd.ch

Accepted for publication 18 December 2001

\begin{abstract}
Background and aims: This was a prospective blinded study to compare computed tomography (CT) colonography, performed with multidetector arrays $\mathrm{CT}$ scan (MDCT), with conventional colonoscopy for the detection of colorectal neoplasia.

Methods: Fifty patients were examined by MDCT after standard bowel preparation and rectal air insufflation in the supine and prone positions. Data sets were examined by one radiologist and one gastroenterologist blinded to the patient's history and colonoscopy results. Patients subsequently underwent colonoscopy on the same day, which served as the gold standard.

Results: Nine of 11 lesions $>10 \mathrm{~mm}(82 \%), 5 / 15$ lesions of $6-9 \mathrm{~mm}(33 \%)$, and $1 / 42$ polyps $<5 \mathrm{~mm}$ $(3 \%)$ were detected by MDCT colonography. One false positive result for a structure larger than $10 \mathrm{~mm}$ was described. Nineteen of 21 patients who had no lesions during conventional colonoscopy were considered free of lesions by MDCT colonography, yielding a per patient specificity of $90 \%$.

Conclusion: MDCT colonography provides good data quality and has good sensitivity and specificity for the detection of colonic lesions of $10 \mathrm{~mm}$ or more.
\end{abstract}

In spite of the initial promising results, CT colonography is not yet ready for general clinical use. ${ }^{16}$ Improvements in this method are still required, especially with regard to data acquisition and quality, and data post-processing software; also, observers require a sufficient learning period. ${ }^{813} 14$

In all published series, data acquisition was performed with single slice helical CT. However, multidetector arrays computed tomography (MDCT) is rapidly entering into clinical use. This new technique has a great potential for clinical application mainly due to an important reduction in scanning time. In our experience, ${ }^{14}$ most false results were due to motion artefacts.

In this report, we have evaluated the performance of MDCT colonography compared with conventional colonoscopy in the first series (to our knowledge) of patients.

\section{METHODS}

The study was approved by the ethics review board of our institution. All patients gave informed written consent prior to entering the study.

\section{Patients}

A total of 51 consecutive patients were enrolled in this prospective study. All patients underwent standard preparation for colonoscopy with 4 litre of methyl-cellulose (Fordtran, Streuli, Switzerland) which was ingested 12 hours prior to the investigations. Indications for colonoscopy were history of prior polyps or colon cancer, unexplained abdominal pain, and iron deficiency anaemia. Patient age ranged between 50 and 75 years. Exclusion criteria were patient refusal or inability to participate.

\section{Image acquisition}

All CT examinations were performed with MDCT scans (LightSpeed; GE, Milwaukee, USA). Prior to scanning, all patients received an intravenous injection of $20 \mathrm{mg}$ of

Abbreviations: $\mathrm{CT}$, computed tomography; MDCT, multidetector arrays computed tomography. 
$N$-butyl-scopolamine (Buscopan; Boehringer Ingelheim, Germany) to reduce bowel spasm during air insufflation and potential motion artefacts during scanning.

All patients underwent rectal room air insufflation on the CT table using a standard enema tube without a balloon cuff. Air insufflation was started in the left lateral position and terminated in the dorsal position. Adequate colonic distension was checked using a scout view, indicating if the entire colon was adequately distended and no segment of the colon was collapsed. After supine data acquisition, most patients (43) were turned to the prone position, seven patients being examined in the left lateral position. Prior to the second acquisition, the scout view was repeated and air insufflation reassessed. If necessary, additional air insufflation was performed (nine patients).

CT scanning was performed in the cranio-caudal direction. Patients were asked to hold their breath during acquisition. If this was not possible, data acquisition was achieved with superficial respiration.

\section{Technical parameters}

Technical parameters for the MDCT examinations were as follows: beam collimation $5 \mathrm{~mm}$, table feed $15 \mathrm{~mm}$ per rotation, and pitch 3:1. Image reconstructions were performed with a slice thickness of $2.5 \mathrm{~mm}$ with reconstruction intervals of $2 \mathrm{~mm}$ (overlap $0.5 \mathrm{~mm}$ ). CT scanning was performed in the supine and prone positions at $90 \mathrm{~mA}$ and $120 \mathrm{kV}$, with a standard algorithm and a $512 \times 512$ matrix size.

All MDCT examinations were followed by conventional colonoscopy (15-45 minutes). During colonoscopy, lesions were photographed, measured, and subsequently removed or biopsies taken. Physicians who performed conventional colonoscopy were not involved in the interpretation of MDCT colonography.

\section{Image processing}

All further data processing and interpretation were performed on a Sun II Spark workstation (Microsystems Mountain View, California, USA). Reconstructed data were stored on optical discs for subsequent evaluation. The software tool used on the workstation was GE Advantage Windows-Navigator, which uses a combination of two dimensional and three dimensional reconstructed and reformatted rendered images. The three dimensional reconstructions are performed with a surface rendering algorithm. This program requires a user defined threshold between the intraluminal air and colon wall for the three dimensional data sets. The angle of vision for the intraluminal views can be interactively modified but in nearly all cases an angle of $60^{\circ}$ was used.

\section{Data interpretation}

One staff radiologist with experience of approximately 60 CT colonography data sets and one gastroenterologist with vast experience in colonoscopy evaluated the MDCT data sets consensually. Both were unaware of the indications and results of colonoscopy. Data interpretation started with the supine axial sections. The observers scrolled through the data sets. This was followed by similar interpretation of the prone or left lateral position images. All lesions identified on two dimensional axial images were systematically re-evaluated or verified on prone or left lateral axial data sets, coronal and sagittal two dimensional and three dimensional reconstructions. Identified lesions were recorded in two dimension and three dimension. Their size and precise location were recorded. Measures were taken in reconstructed two dimensional images. Time required for data interpretation was recorded.

The quality of prone and supine MDCT was assessed. Good quality was defined as good distension of the bowel wall allowing assessment of the whole bowel wall. Sufficient quality implied suboptimal distension, allowing 90-95\% bowel
Table 1 Sensitivity of multidetector arrays computed tomography (MDCT) colonography for lesions according to size

\begin{tabular}{lllr}
\hline & $\begin{array}{l}\text { No of lesions } \\
\text { found by } \\
\text { conventional } \\
\text { colonoscopy }\end{array}$ & $\begin{array}{l}\text { No of lesions } \\
\text { found by MDCT } \\
\text { colonography }\end{array}$ & Sensitivity \\
\hline Lesion $\leqslant 5 \mathrm{~mm}$ & 41 & 1 & $4 \%$ \\
Lesion 6-9 mm & 15 & 5 & $33 \%$ \\
Lesion $\geqslant 10 \mathrm{~mm}$ & 11 & 9 & $82 \%$ \\
\hline
\end{tabular}

evaluation. Insufficient quality was insufficient bowel distension and/or less than $90 \%$ bowel wall displayed and accessible to data evaluation.

Polyp detection was assessed on a per polyp basis. Polyps were classified according to their size, as described in conventional colonoscopy: lesions $\geqslant 10 \mathrm{~mm}, 6-9 \mathrm{~mm}, \leqslant 5 \mathrm{~mm}$. Specificity was assessed on a per patient basis - that is, the patient was considered as free of colon lesions or having a colonic lesion, independent of the size, histology (carcinoma, adenomatous or hyperplastic polyp), or number of lesions.

\section{RESULTS}

Fifty one patients were included in the study. Age range was 50-75 years. One patient was excluded from the study as she did not follow the instructions for bowel preparation necessary for colonoscopy. MDCT and colonoscopy were successfully performed in all remaining 50 patients. No complication was observed using either technique. No patient required sedation for MDCT scanning.

Thirty six patients were able to hold their breath during data acquisition. For the remaining 14 patients, data acquisition was achieved with superficial respiration. Mean scanning time for supine, prone, or left lateral data acquisition was 24 seconds. Mean time the patient remained in the CT suite was 17 minutes (explanation of the examination procedure, installation on the table, air insufflation, data acquisition).

Thirty nine patients $(78 \%)$ had good preparation, seven (14\%) sufficient preparation, and four ( $8 \%$ ) insufficient bowel preparation.

At conventional colonoscopy, 67 lesions were found (table 1). Forty one lesions were $\leqslant 5 \mathrm{~mm}, 15$ were $6-9 \mathrm{~mm}$ (fig 1 ), and 11 were $\geqslant 10 \mathrm{~mm}$ (fig 2 ). Nine of 11 lesions $\geqslant 10 \mathrm{~mm}$ were found at MDCT colonography (82\%), 5/15 lesions of 6-9 mm (33\%), and $1 / 41$ lesions of $\leqslant 5 \mathrm{~mm}(4 \%)$.

In a retrospective analysis concerning missed lesions, two lesions $\geqslant 10 \mathrm{~mm}$ were detected retrospectively in the data sets. For the first 25 patients, the observers detected all five polyps $\geqslant 10 \mathrm{~mm}$; two polyps of this size were missed between patient Nos 26 and 50.

Seven of 10 missed lesions of 6-9 mm were detected retrospectively. Three were pedunculated polyps (two of $8 \mathrm{~mm}$, one of $7 \mathrm{~mm}$ ) and four were sessile polyps (one polyp of $8 \mathrm{~mm}$, two of $7 \mathrm{~mm}$, and one of $6 \mathrm{~mm}$ ). Concerning polyps $\leqslant 5 \mathrm{~mm}, 18$ were found retrospectively in the data sets. Among the 22 that could not be detected retrospectively, 12 were localised in the rectosigmoid, seven in the caecum and ascending colon, two in the transverse colon, and one in the descending colon.

During examination of patient Nos 1 to 25, conventional colonoscopy detected five polyps $\geqslant 10 \mathrm{~mm}$. All five were detected by CT colonography. During examination of patient Nos 26 to 50, conventional colonoscopy detected six polyp $\geqslant 10$ $\mathrm{mm}$. Four were found by CT colonography.

Twenty one patients had no lesions at conventional colonoscopy. Nineteen were considered free of lesions at MDCT colonography, giving a specificity per patient of $90 \%$. 

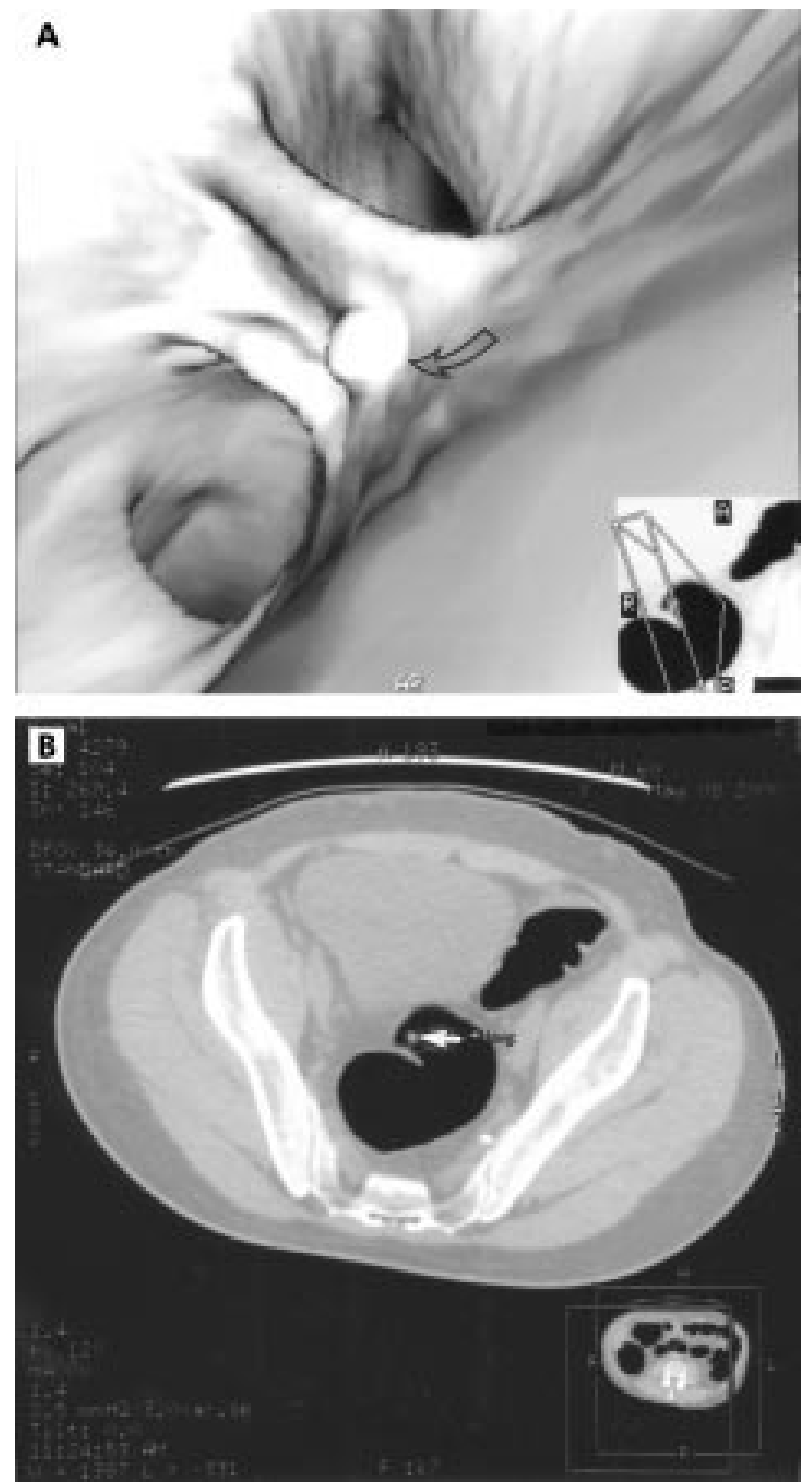

Figure 1 Three dimensional image of an $8 \mathrm{~mm}$ pedunculated rectal polyp $(A$, arrow); corresponding axial computed tomography image ( $B$, arrow).

One $11 \mathrm{~mm}$ structure was seen at MDCT colonography but not identified at conventional colonoscopy. This structure was assumed to be a false positive. It was localised in a spastic region of the sigmoid colon of a patient with insufficient bowel preparation.

Median time required for data interpretation was 13 minutes (range 7-18). Evaluation of supine and prone two dimensional data sets required an average of 10 minutes. Three minutes on average were necessary to verify suspect structures with supplemented three dimensional endoluminal images.

\section{DISCUSSION}

We have described the diagnostic performance and potential of CT colonography, carried out with multidetector CT, to identify space occupying lesions of the colon. To our knowledge, there are no published studies comparing CT colonography, performed entirely with multidetector arrays helical CT, with conventional colonoscopy.

In a previous study based on a "single" detector array helical CT scanner, the results concerning sensitivity and specificity were mediocre. ${ }^{14}$ This was mainly due to false negatives
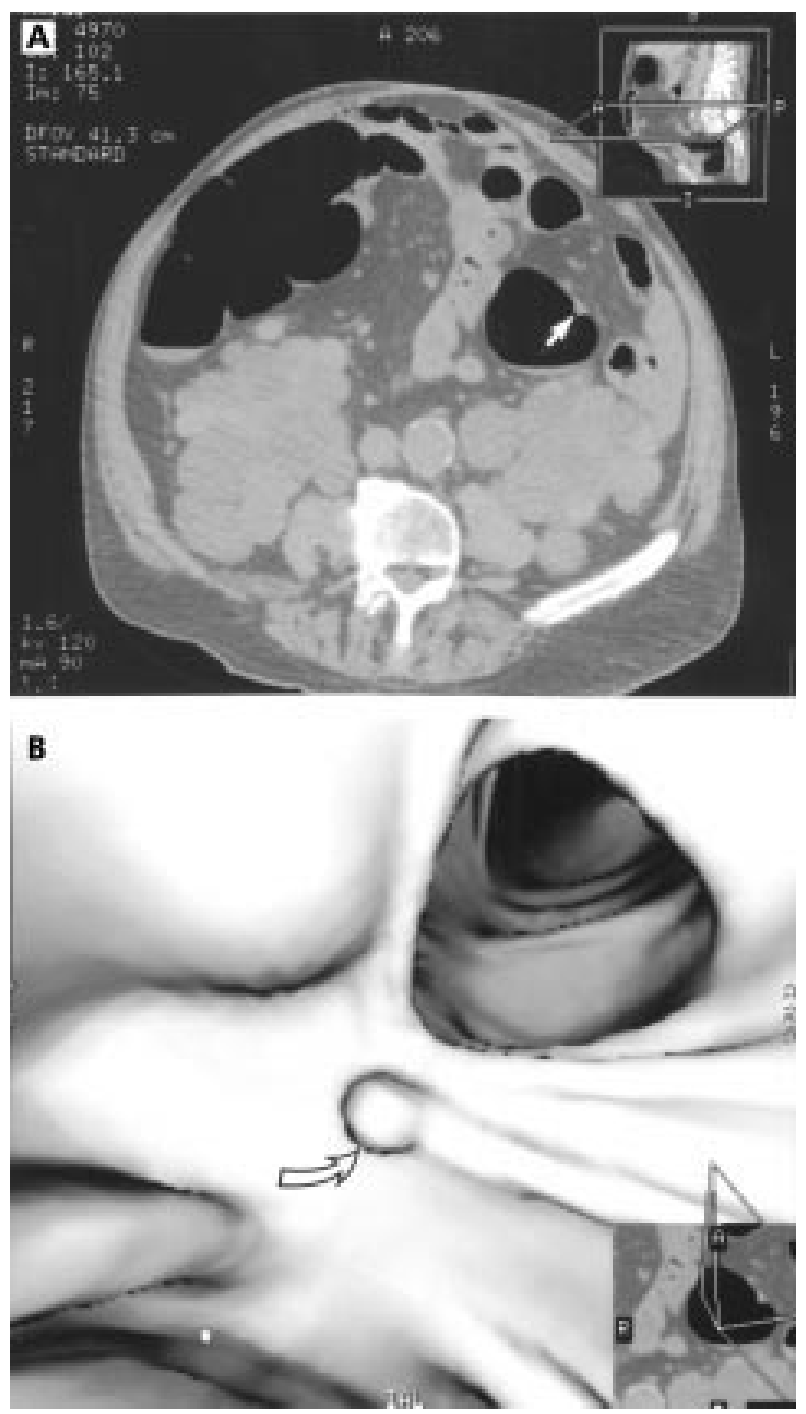

Figure 2 Transverse computed tomography image of a $10 \mathrm{~mm}$ sessile polyp of the descending colon ( $A$, arrow); corresponding three dimensional image ( $B$, arrow).

findings related to the poor data quality because of motion artefacts, residual fluid, and retained faecal material. In the present study, sensitivity for the detection of lesions of $\geqslant 10$ mm was $82 \%$ compared with $52 \%$ in the previous study. Several reasons may be responsible for this improvement.

In the previous study, based on 50 patients, only 11 (22\%) had good preparation (that is, more than $95 \%$ of the bowel wall could be assessed), 25 (50\%) had sufficient (that is, 90-95\% of the bowel could be assessed), and 14 (28\%) had insufficient (less than $90 \%$ of the bowel wall could be assessed) bowel preparation. The quality of bowel preparation improved in the present study: 39 patients $(78 \%)$ had good, seven (14\%) sufficient, and only four patients ( $8 \%$ ) had insufficient bowel preparation.

The potential of the MDCT scanner lies in the possibility of faster data acquisition and improved spatial resolution. In this study, the whole abdomen could be scanned in an average time of 24 seconds. This is far less compared with the single slice CT scanner (approximately 35 seconds) and results in less motion artefacts due to respiration and bowel movements.

Other than better bowel preparation and fewer artefacts due to faster scanning, ${ }^{17}$ other factors may have influenced the increased sensitivity: the combination of supine and prone acquisition improves sensitivity by approximately $15 \% .{ }^{19}$ 
Another contributing factor may be the increased experience of the interpreters gained from the previous study. ${ }^{14}$

The limitations and pitfalls of the method are illustrated by the following observations: two of 11 lesions larger than 10 $\mathrm{mm}$ were missed. Both were retrospectively found in the MDCT data sets. One was a flat polyp localised at the ileocaecal valve (fig 3 ). Flat lesions have a height that does not exceed one third of the size of the base (Fidler JJ, et al. Radiology 2000;217(P);582). Flat polyps are more likely to be missed than sessile ones as they do not, or only slightly, alter the colonic contour. However, they have a clearly different soft tissue attenuation compared with fat (Glücker T, et al. SRG Meeting Scottsdale, Arizona, 2001). This lesion was interpreted as a large lipomatous ileocaecal valve. Retrospectively, this lesion was easily found on two dimensional axial data sets as it represented thickening of the caecum wall. The lesion was far less evident on three dimensional data sets. Hence this lesion was missed because of a perceptive error and was not due to technical failure. According to our limited experience, the ileocaecal region proved to be a difficult area to screen because of the converging folds. Another factor is that the ileocaecal valve appeared to be lipomatous in several patients. This can easily give rise to misinterpretations on three dimensional images. The second missed lesion greater than $10 \mathrm{~mm}$ was a $10-15 \mathrm{~mm}$ polyp. It was localised on a fold on the superior and anterior aspect of the hepatic flexure. This polyp was missed on two dimensional and three dimensional data sets but retrospectively identified on two dimensional sagittal views

In this study, CT colonography had a poor performance for lesions of 6-9 mm. Ten of 15 lesions with a diameter of 6-9 $\mathrm{mm}$ were initially missed on MDCT colonography. Among these, seven were found retrospectively and must be considered perceptive errors. They were three pedunculated and four sessile polyps. Pedunculated polyps have a stalk and can significantly alter their position when the patient is scanned in two different positions. ${ }^{15}$ Awareness of this fact and further training of readers in CT colonography may improve readers' performances for lesions of this size. Three missed polyps at MDCT colonography could not be found retrospectively in MDCT data sets and were considered as technical errors: two were localised in collapsed areas. One sessile polyp measuring $6 \mathrm{~mm}$ could not be found retrospectively in the MDCT data sets.

Sensitivity for lesions smaller than $5 \mathrm{~mm}$ was only $4 \%$ - that is, only one among 41 small polyps was detected. Retrospectively, 18 of the 40 missed small polyps were detected. Most polyps smaller than $5 \mathrm{~mm}$ that could not be detected were localised in the rectosigmoid and caecum. Both areas are characterised by a complex anatomical structure with converging folds. Altered scanning techniques with beam collimation less than $5 \mathrm{~mm}$ and a reconstruction interval of $1 \mathrm{~mm}$ or $1.25 \mathrm{~mm}$ could improve resolution and thus increase detection of smaller polyps if clinically indicated. ${ }^{19}$ However, this would significantly increase the radiation dose for the patient. There is no consensus as to whether an increased detection rate for polyps smaller than $5 \mathrm{~mm}$ is necessary. Only about $5 \%$ of lesions smaller than $10 \mathrm{~mm}$ contain high grade dysplasia $^{20}{ }^{21}$ and only $1.3 \%$ of polyps smaller than $10 \mathrm{~mm}$ are malignant. ${ }^{22}{ }^{23}$ In the current literature there is no general consensus concerning the clinical relevance of small polyps and the minimal size of polyps that should be targeted by screening methods. ${ }^{21-24}$ As demonstrated in the retrospective data analysis, CT colonography in this series with a multidetector CT scanner had the technical ability to detect all lesions $\geqslant 10 \mathrm{~mm}, 80 \%$ of lesions of $6-9 \mathrm{~mm}$, and approximately $47 \%$ of lesion $<5 \mathrm{~mm}$.

The weak point of the study concerns conventional colonoscopy, considered as a suboptimal gold standard. Indeed, several studies have indicated that conventional colonoscopy has a rate of missed lesions of up to $24 \%{ }^{25}$ In $10 \%$ of patients, the caecum cannot be visualised. ${ }^{26}$
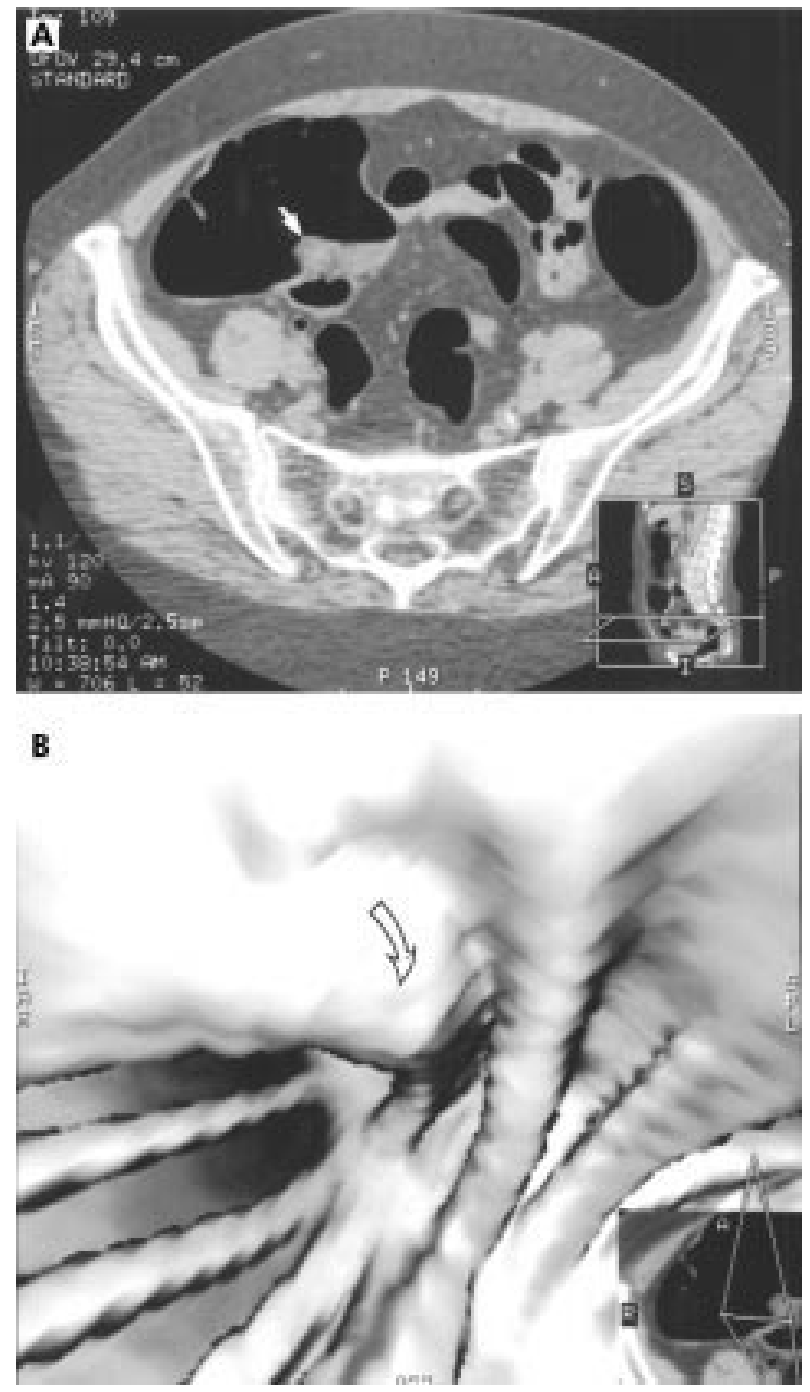

Figure 3 Missed polyp of the ileocaecal valve. The lesion can be misinterpreted as stool or a prominent ileocaecal valve ( $A$, arrow). The lesion is difficult to capture on three dimensional image (B, arrow)

Our study was performed with readers having only a limited previous experience of CT colonography with data sets of approximately 60 patients. Further training was mainly obtained by review of previously missed lesions. In the course of the study, at least for polyps larger than $10 \mathrm{~mm}$, no significant change in the rate of polyp detection was seen. This may be related to the small number of polyps (only 11 ) larger than $10 \mathrm{~mm}$ in this series. The readers did not recognise all of the lesions demonstrated by multidetector CT scanner. Further training is necessary to obtain the best yield from the possibilities offered by colonography performed with multidetector CT scanners.

CT colonography, performed with either the "single slice" or now with the multislice helical CT, is a full structural colonic examination that is fast, dose not require sedation, is non-invasive, and also offers the possibility of detecting significant extracolonic findings. ${ }^{27}$ However, CT colonography is dependant on good bowel preparation, such as conventional colonoscopy. Poor bowel preparation can result in erroneous conclusions as retained stool material can either mask colon polyps or mimic polyps or masses. ${ }^{15}$ Excess fluid remaining from the bowel preparation can also mask colonic lesions. Attempts to minimise the deleterious effects of residual stool and fluid can be undertaken by scanning in different positions 
(to move stool and fluid) whereas during conventional colonoscopy excess fluid can be aspirated.

In conclusion, MDCT offers good data quality with supine and prone data sets, thus assuring significant sensitivity per polyp and specificity per patient. CT colonography will benefit from further improvements in imaging techniques, computer software, and observer experience. In our opinion, MDCT is an important improvement in the "hardware", permitting a clear improvement in data quality of MDCT colonography compared with single detector array $\mathrm{CT}$, and warrants further investigation. Further indications for CT colonography other than screening patients with a high or moderate risk of colonic neoplasms or incomplete colonoscopy may be detection of primary lesions for patients with known liver metastases.

\section{ACKNOWLEDGEMENT}

We would like to thank Mr JG Fletcher MD, Mayo Clinic Rochester, for his help with this manuscript

\section{Authors' affiliations}

Th Gluecker, Department of Diagnostic and Interventional Radiology, University Hospital CHUV Lausanne, Switzerland, and Department of Radiodiagnostic, Mayo Clinic Rochester, Minnesota, USA

G Dorta, W Keller, P Jornod, Department of Gastroenterology and Hepatology, University Hospital CHUV Lausanne, Switzerland R Meuli, P Schnyder, Department of Diagnostic and Interventional Radiology, University Hospital CHUV Lausanne, Switzerland

\section{REFERENCES}

1 Wingo PA, Tong T, Bolden B. Cancer Statistics 1995. Cancer J Clin 1995;45:8-13

2 Stryker SJ, Wolff BG Culp CE, et al. Natural history of untreated colonic polyps. Gastroenterology 1987;93:1009-13.

3 Winawer SJ, Fletcher, RH Miller L, et al. Colorectal cancer screening: clinical guidelines and rationale. Gastroenterology 1997;1 1:594-642.

4 Eddy DM. Screening for colorectal cancer. Ann Intern Med 1990:113:373-84

5 Liebermann DA, Smith FW. Screening for colon malignancy with colonoscopy. Am J Gastroenterol 1991;86:946-51.

6 Hara AK, Johnson CD, Reed JE, et al. Detection of colorectal polyps by computed tomographic colonography: feasibility of a novel technique. Gastroenterology 1996;110:284-90.
7 Hara AK, Johnson CD, Reed JE, et al. CT colonography: initial assessment of sensitivity and specificity. Radiology 1997;205:59-65.

8 Johnson CD, Ahlquist DA. Computed tomography colonography (virtual colonoscopy): a new method for colorectal screening. Gut 1999:44:301-5.

9 Hara AK, Johnson CD, Reed JE, et al. Colorectal polyp detection with CT colonography: two- versus three-dimensional techniques. Radiology 1996;200:49-54

10 Vining DJ. Virtual endoscopy: is it reality? Radiology 1996;200:30-1.

11 Fenlon HM, Nunes DP, Schroy PC, et al. A comparison of virtual and conventional colonoscopy for the detection of colorectal polyps. N Engl J Med 1999;341:1496-503.

12 Kay CL, Kulling D, Hawes RH, et al. Virtual endoscopy-comparison with colonoscopy in the detection of space-occupying lesions of the colon. Endoscopy 2000;32:226-32.

13 Rex DK. Virtual colonoscopy: time for some tough questions for radiologists and gastroenterologists. Endoscopy 2000;32:260-3.

14 Pescatore P, Gluecker T, Delarive J, et al. Diagnostic accuracy and interobserver agreement of CT colonography (virtual colonoscopy). Gut 2000;47:126-30

15 Fletcher JG, Johnson CD, MacCarthey, et al. CT colonography: potential piffalls and problem solving techniques. AJR 1999;172:1271-

16 Bond JH. Virtual Colonoscopy-promising, but not ready for widespread use. N Engl J Med 1999;341:1540-2.

17 Fletcher JG, Johnson CD. Computer tomographic colonography: current and future status for colorectal cancer screening. Semin Roentgenol 2000:35:385-93.

18 Fletcher JG, Johnson CD, Welch TJ, et al. Optimization of CT colonography technique: prospective trial in 180 patients. Radiology 2000;216:704-11

19 Rogalla P, Meiri N, Rueckert JC, et al. Colonography using multislice CT. Eur J Radiol 2000;36:81-5.

20 Hermanek P. Dysplasia-carcinoma sequence, types of adenoma and early colorectal carcinoma. Eur J Surg Oncol 1987;13:141-3.

21 Shinya H, Wolff Wl. Morphology, anatomic distribution and cancer potential of colonic polyps. Ann Surg 1979;190:679-83.

22 Morson BC. The polyp cancer sequence in the large bowel. Proc $R$ Soc Med 1974;74:451-7.

23 Muto T, Bussey HJR, Morson BC. The evolution of cancer of the colon and rectum. Cancer 1975;36:2251-70.

24 Hofstad B, Vatn MH, Andersen I, et al. Growth of colorectal polyps and evaluation of unresected polyps for a period of three years. Gut 1996;39:449-56.

25 Rex DK, Cutler CS, Lemmel GT, et al. Colonoscopic miss rates of adenomas determined by back to back colonoscopy. Gastroenterology1997:112:24-8.

26 Ferruci JT. Screening for colon cancer: programs of the American College of Radiology. Am J Roentgenol 1993;160:999-1003.

27 Hara AK, Johnson CD, MacCarthy, et al. Incidental extracolonic findings at CT colonography. Radiology 2000;215:353-7. 